\title{
General Theory for Light Propagation and the Motion of Bodies with Mass
}

\author{
Daniel LLARENS \\ 38 Street \#1284, La Plata, 1900, Argentina \\ daniel.llarens[at]grupome.com
}

\begin{abstract}
A. Einstein presented to the scientific world the so-called Theory of Relativity ((1905) Special Relativity Theory, (1916) General Relativity Theory). One of the essential aspects of this theory has become the holy grail of modern physics: "The speed of light in the vacuum is constant". In the last century, this concept caused passionate controversies in the scientific world for its implications on how the universe works, its origin, and its possible end. So the idea that the speed of light can be variable, and consequently the Theory of Relativity not correct, currently it is considered problematic because this idea perhaps fails to explain the wide range of modern tests of general relativity, like the dynamics of the Binary Pulsar and the detection of gravitational waves. The observations of Edwin Powell Hubble (1929) allowed determining other characteristics of light propagation. Hubble observed that the light that reaches us from distant stars has spectral lines that are red-shifted all the more the far-away the star is from us. The Theory of Relativity along with Hubble's observations laid the formulations for another conclusion that marked our current understanding of how the universe works: the universe had an origin, called the big-bang, approximately 13.5 billion years ago. The recent launch of the James Webb Space Telescope has as one of its main objectives to observe the birth of the universe. The conclusion that the universe has had a beginning may perhaps be the result of a conception of how we see nature, everything we know has a beginning and an end! The conclusion that the universe has also had a beginning may be wrong and therefore we can see the need to explain the existence of an infinite universe that has no beginning and will have no end. This article proposes a new theory that explains how the universe works where the observations concerning the "red-shift" are explained by a new property of light called "SLIDING" which increases the wavelength of light. The wavelength increases are only significant over very large propagation distances (million light-years), which is why the change in the wavelength was not observed in the Michelson/Morley experiments. The increase in the wavelength is done without changes in the photon energy. The proposed new property of our universe called "SLIDING" also affects bodies in motion. In proof of the aforementioned, in this article, it is demonstrated that SLIDING can explain the perihelion's precession in the orbits of the planets. As a result of the proposed theory, the universe did not have an event that we can call as it birth, it has always existed and will exist forever. The Olbers's paradox is avoided because the light that reaches us from distant stars has a wavelength in the infrared.
\end{abstract}

Keywords: Relativity, Newtonian gravitation, Redshifted, Light speed

\section{Introduction}

Experiments conducted in 1887 by Albert Abraham Michelson and Edward Morley showed that the speed of light in the vacuum $\left(\mathrm{c}_{0}\right)$ is constant and independent of the speed at which the source of light emission moves, that is, for light the addition of velocities resulting from Newton's Laws for bodies in motion is not applicable.

The observations of Edwin Powell Hubble (1929) allowed determining other characteristics of light propagation. Hubble observed that the light that reaches us from distant stars has spectral lines that are red-shifted all the more the far-away the star is from us.

The phenomenon of increase in wavelength observed by Hubble has been explained by assuming that the so-called Doppler effect manifests itself in the propagation of light, as well as what happens in the propagation of waves in classical mechanics. The redshift is explained by the fact that the light source (distant star) is moving away from us at a higher speed (v) when far away the star is from us.

Taking as an axiom that a light beam that propagates in the vacuum has a constant propagation speed $\left(\mathrm{c}_{0}\right)$ and that no bodies with mass can move faster than light speed, Einstein develops the so-called "Theory of Relativity (TR)" published in the years 1905 and 1916. The Einstein's universe is significantly more complex than Newton's universe among other things by i) proposing that "time" is a variable that depends on the motion of the frame of reference where the observer is, and ii) that the Law of Universal Gravitation is explained as a deformation of "space-time" by the effect of the gravitational field of the stars.

Newton's Laws allowed determining the movement of planets around the sun. However, the Gravity Force (FG) resulting from the Law of Universal Gravitation cannot fully explain the movement of the planets. This is observed when analyzing the orbit of Mercury characterized by a significant perihelion's precession. Similar, but minor, effects are observed in the orbits of Venus and Earth ${ }^{1}$.

According to the TR, the precession angle $(\delta)$ for elliptical orbits can be determined by the following expression (it is assumed that the mass of the planets is much smaller than the mass (M) of the sun)

$$
\delta=\frac{3 \times 2 \pi}{(1+\varepsilon) R_{P}} \times \frac{G M}{c_{0}^{2}}
$$

Where:

$$
\begin{aligned}
& \varepsilon \text { : Eccentricity } \\
& \mathrm{R}_{\mathrm{p}} \text { : radius at perihelion }
\end{aligned}
$$

The experiments carried out in recent years seem to confirm the consistency of the observed facts with the predictions of

\footnotetext{
${ }^{1}$ The measured precession values are (arcsec/cen): Mercury: 43.11 \pm 0.45 ; Venus; $8.4 \pm 4.8$; Earth:5.0 \pm 1.2 - The

Astronomical Journal. 1956.
}

\section{Volume 10 Issue 12, December 2021}




\section{International Journal of Science and Research (IJSR)}

ISSN: 2319-7064

SJIF (2020): 7.803

the TR. However, this may be a tautological vision of reality since it cannot be concluded that the TR is the only one that allows explaining the observed facts.

To explain the observed facts above described, in this document a new theory is developed to explains the motion of bodies and the propagation of light in vacuum.

Within the framework of the proposed theory, the motion of a body is explained by a new characteristic of the universe that produces that the distance traveled for a body in a time interval $(\Delta \mathrm{t})$ is greater than the ones determined by Newton's Laws.

$$
\begin{gathered}
\Delta x=v_{M} \times \Delta t \\
v_{M}=\frac{v}{1-\frac{v^{2}}{c_{0}^{2}}+\left(\frac{v^{2}}{c_{0}^{2}}\right)^{2}} \geq v
\end{gathered}
$$

In the same way, light has a new property: its wavelength increases with the distance (D) traveled by the light beam even when the light source is at rest within the inertial system where is located the observer. The proposed increase in wavelength is made without changes in the energy of the photons ( $\mathrm{f}=$ constant).

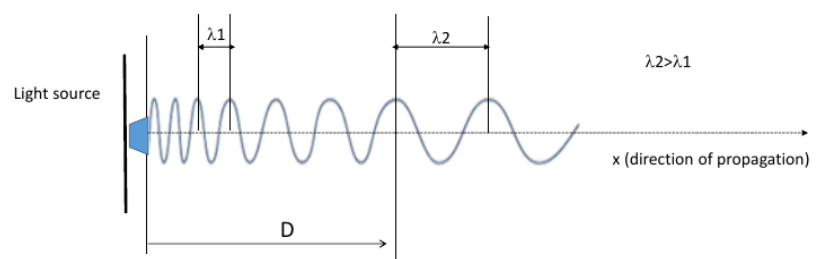

Figure 1: Theory about light propagation. Wavelength increases with the propagation distance

Next, the theoretical aspects associated with the propagation of light and the motion of bodies with mass are developed.

As a conclusion, it is demonstrated that the propagation of energy through the vacuum, either in the form of a light beam or a moving body with mass $(\mathrm{m})$, produces the socalled "SLIDING EFFECT" which is responsible for the two physical phenomena commentated above.

\section{SLIDING Effect}

The theory proposed considers that if a constant force $(F)$ is applied to a body with mass (m), the resulting acceleration (a) is determined by the following differential equation:

$$
\begin{gathered}
\dot{a}=\frac{F}{m} \\
a=\dot{a} \times\left(1-\frac{v^{2}}{c^{2}}\right) \\
v=c \times \sqrt{1-\frac{a}{\dot{a}}} \\
\int a d t=c \times \sqrt{1-\frac{a}{\dot{a}}} \\
a=c \times \frac{d}{d t} \sqrt{1-\frac{a}{\dot{a}}}
\end{gathered}
$$

$$
\begin{gathered}
a=\frac{c}{2} \times \frac{1}{\sqrt{1-\frac{a}{\dot{a}}}} \times\left(-\frac{1}{\dot{a}} \times \frac{d a}{d t}\right) \\
\frac{d a}{d t}=-2 \frac{a \times \dot{a}}{c} \sqrt{1-\frac{a}{\dot{a}}} \\
a(t=0)
\end{gathered}
$$

The velocity (v) results from Newton's law.

$$
\frac{d v}{d t}=a
$$

The next figure shows the resulting variation of acceleration $\mathrm{a}(\mathrm{t})$ considering $(\mathrm{F} / \mathrm{m}=5)$. As can be seen, the SLIDING effect produces that the acceleration (a) decreases with time even when the force applied to the body is constant. Acceleration (a) tends to zero when speed (v) tends to light speed $\left(\mathrm{c}_{0}\right)$. The maximum speed $(\mathrm{v})$ is equal to $\left(\mathrm{c}_{0}\right)$.

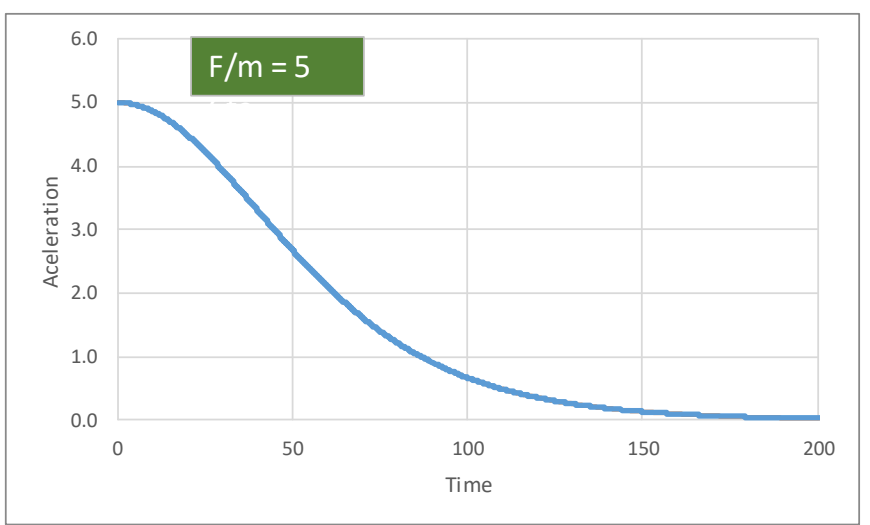

Figure 2: Example. Aceleration (a) for $\mathrm{F} / \mathrm{m}=\mathrm{Cte}$



Figure 3: Example. Velocity (v) for $F / m=C t e$

The SLIDING effect produces that the body travels a distance $(\Delta \mathrm{x})$ in a time interval $(\Delta \mathrm{t})$ that is grated that the ones calculated by Newton's law.

$$
\frac{d x}{d t}=v_{M}=\frac{v}{1-\frac{v^{2}}{c_{0}^{2}}+\left(\frac{v^{2}}{c_{0}^{2}}\right)^{2}} \geq v
$$

The speed $v_{M}$ is higher than ( $\left.v\right)$. The maximum value is 1.0666 of light speed $\left(c_{0}\right)$ that is reached when $\mathrm{v}=0.876 \mathrm{x} \mathrm{c}_{0}$. 




Figure 4: Measure Velocity $\left(\mathrm{v}_{\mathrm{M}}\right)$

The total energy (ET) of a mass that is moving to a speed (v) is equal to the sum of its rest energy $\left(E_{0}\right)$ and the Kinetic Energy $\left(\mathrm{E}_{\mathrm{K}}\right)$.

$$
E T=E_{0}+E_{K}=m c_{0}^{2}+\frac{1}{2} m \frac{v^{2}}{\left(1-\frac{v^{2}}{c_{0}^{2}}\right)^{2}}
$$

The Kinetic Energy (Ek) tends to infinity when speed (v) tends to the light speed $\left(\mathrm{c}_{0}\right)$. This means that a body with mass never could travel at speed $(v)$ higher than $\left(\mathrm{c}_{0}\right)$.

As a result of the proposed theory, a body in motion have two velocities: i) the velocity (v) that determines the Kinetic Energy of the body with a maximum value equal to light speed (c), and ii) the velocity $\left(\mathrm{v}_{\mathrm{M}}\right)$ that determines the distance traveled by the body that can be higher than $\left(\mathrm{c}_{0}\right)$. An observer at rest, in the same inertial system as the moving body, will measure velocity $\left(\mathrm{v}_{\mathrm{M}}\right)$.

The conclusions above indicated are valid in the vacuum faraway from massive stars. Future research should conclude if $\left(\mathrm{v}_{\mathrm{M}}\right)$ is affected by gravitational fields.

\subsection{Uniform Rotational Motion}

For the particular case of a Uniform Rotational Motion with radius (R) and angular velocity $(\omega)$, the SLIDING effect produces an advance $(\delta)$ (precession), which is added to the angular velocity:

$$
\begin{gathered}
\frac{d \dot{\phi}}{d t}=\omega_{M}=\frac{\omega}{1-\frac{v^{2}}{c_{0}^{2}}+\left(\frac{v^{2}}{c_{0}^{2}}\right)^{2}} \\
\omega=\frac{v}{R}
\end{gathered}
$$

Integrating

$$
\begin{gathered}
\dot{\phi}=\int_{0}^{2 \pi} \frac{1}{1-\frac{v^{2}}{c_{0}^{2}}+\left(\frac{v^{2}}{c_{0}^{2}}\right)^{2}} d \phi=2 \pi+\delta \\
\delta=2 \pi \times\left(\frac{1}{1-\frac{v^{2}}{c_{0}^{2}}+\left(\frac{v^{2}}{c_{0}^{2}}\right)^{2}}-1\right)
\end{gathered}
$$

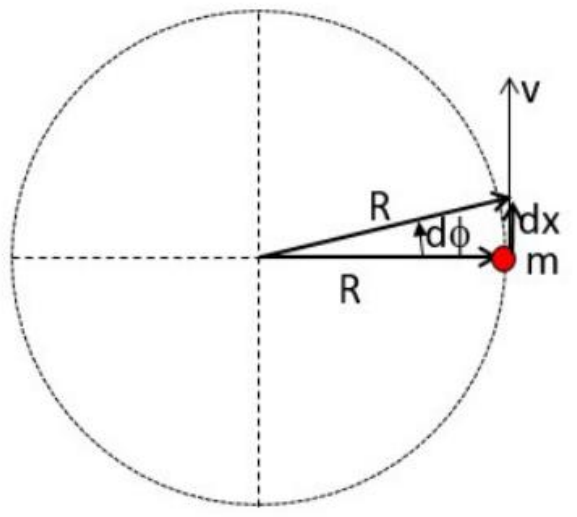

Figure 5: Rotational Motion

If $\left(\mathrm{v}<<\mathrm{c}_{0}\right)$

$$
\delta=2 \pi \times \frac{v^{2}}{c_{0}^{2}}
$$

If $\left(\mathrm{v} \rightarrow \mathrm{c}_{0}\right)$

$$
\delta=0.0
$$

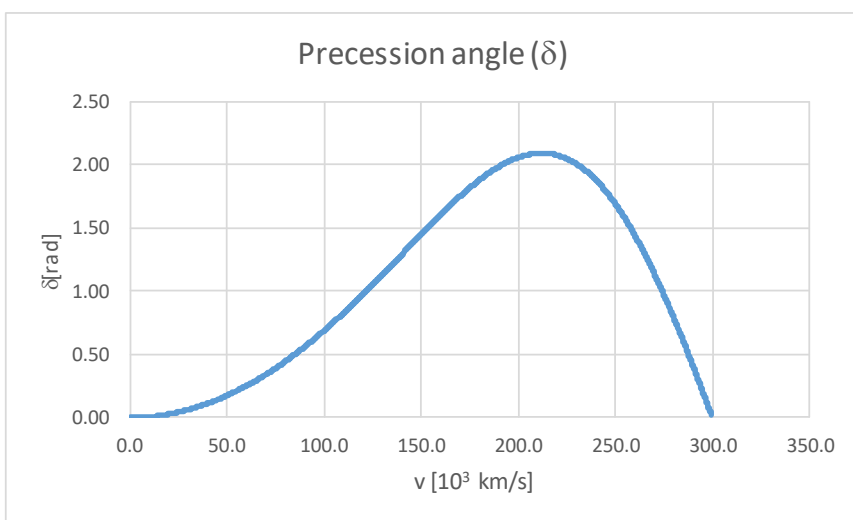

Figure 6: Uniform Rotational Motion. Precession angle

That is, a body with a mass (m) that moves with a uniform rotational motion experiment a precession $(\delta)$ given by the previous expression. The precession is observed as an advance of the body over the orbit so that if its position is photographed every period $(T=2 \pi / \omega)$ it will be observed that the body is not in the same position, having advanced an angle $(\delta)$ in each turn.

\section{Experimental test.}

If the proposed theory about the so-called "Sliding" effect is true, it could be verified by observing the movement of all bodies with mass. For the effect of "SLIDING" to be "observably" experimentally, conditions where the speed (v) of the body is comparable to the speed of light $\left(\mathrm{c}_{0}\right)$, should be achieved.

Experiments like this are those that are carried out using protons in the CERN Large Hadron Collider (LHC) built on the border between France and Switzerland. The main ring of the LHC, with a radius $(\mathrm{R}=27 \mathrm{~km})$, allows accelerating protons to speeds close to the light resulting in an energy of $3500 \mathrm{GeV}\left(\mathrm{ET}^{\mathrm{E}} \mathrm{E}_{0}=3730.3\right)$.

\section{Volume 10 Issue 12, December 2021}


Considering the Total Energy (ET) resulting from equation (5), to achieve a total energy of $3500 \mathrm{GeV}$, the two speed of the proton $\left(\mathrm{v}, \mathrm{v}_{\mathrm{M}}\right)$ should be equal to $\mathrm{v}=0.99423, \mathrm{v}_{\mathrm{M}}=1.00567$ of the speed of light

In the CERN laboratory, it is also possible to test the maximum angular velocity reached by the protons. According to the proposed theory, the minimum elapsed time (Tm) to complete a turn to the LHC ring $(\mathrm{R}=27 \mathrm{~km})$ is equal to:

$$
\operatorname{Tm}[s]=\frac{2 \pi \times 27000}{1.0666 \times 3 \times 10^{8}}=530 \times 10^{-6}
$$

These results can be tested at CERN, which should allow the proposed theory to be verified directly.

\subsection{Perihelion's precession of the planets}

In the case of a planet that moves in its orbit around the sun as a result of the conservative forces (Force of Gravity, Centrifugal Force) the phenomenon of "SLIDING" is also manifested, resulting in a perihelion's precession $(\delta)$ of its orbit.

In this case, to calculate the precession angle $(\delta)$ it is necessary to take into account the dependence between the radius ( $\mathrm{R}$ ) and the velocity (v) by the balance between the Force of Gravity and the Centrifugal Force.

$$
\begin{gathered}
\frac{G M m}{R^{2}}=m \frac{v^{2}}{R} \\
\omega=\frac{v}{R}=\frac{v^{3}}{G M} \\
\frac{d \delta}{d t}=\frac{d \omega}{d v} \Delta v \\
\frac{d \omega}{d v}=3 \frac{v^{2}}{G M}=\frac{3}{R}
\end{gathered}
$$

If $\mathrm{v}<<\mathrm{c}$

Integrating

$$
\begin{gathered}
\Delta v=\hat{v}-v=v \times\left(\frac{1}{\left(1-\frac{v^{2}}{c^{2}}\right)}-1\right) \\
\Delta v=v \times \frac{v^{2}}{c^{2}} \\
\frac{d \delta}{d t}=\frac{3}{R} \mathrm{v} \times \frac{v^{2}}{c^{2}}=3 \omega \times \frac{v^{2}}{c^{2}}
\end{gathered}
$$

$$
\begin{aligned}
& \delta=\int_{0}^{2 \pi} 3 \omega \times \frac{v^{2}}{c^{2}} d t \\
& \delta=2 \pi \times 3 \frac{v^{2}}{c^{2}}
\end{aligned}
$$

The above equation (6) for the perihelion's precession angle $(\delta)$ is equal the value resulting from the TR (equation (1)) considering a circular orbit $\left(\varepsilon=0 ; R_{p}=R\right)$, so this demonstrates that the "SLIDING" effect can also explain the precession observed for circular orbits.

The precession angle $(\delta)$ for elliptic orbits can be calculated considering the following differential equations:

$$
\begin{gathered}
\frac{d R}{d t}= \pm \sqrt{2\left(E T+\frac{G M}{R}-\frac{1}{2} \times \frac{L^{2}}{R^{2}}\right)} \\
\frac{d \emptyset}{d t}=\frac{L}{R^{2}} \times \frac{\left(1+2 \frac{v^{2}}{c^{2}}\right)}{\left(1-\frac{v^{2}}{c^{2}}\right)}
\end{gathered}
$$

Where:

$$
\begin{gathered}
L=\frac{v_{p} R_{p}}{\left(1-\frac{v_{p}^{2}}{c_{0}^{2}}\right)} \\
E T=-\frac{G M}{R_{p}}+\frac{1}{2} \times \frac{L^{2}}{R_{p}^{2}} \\
v_{p}^{2}=\frac{G M}{R_{p}} \times(1+\varepsilon) \\
v^{2}=2 G M\left(\frac{1}{R}-\frac{(1-\varepsilon)}{2 R_{p}}\right) \\
v \ll c \\
\mathrm{M} ; \mathrm{R}_{\mathrm{p}}, \varepsilon: \text { Data }
\end{gathered}
$$

At perihelium

$$
\begin{gathered}
\frac{d R}{d t}=0.0 \\
\delta=\emptyset\left(t_{2}\right)-\emptyset\left(t_{1}\right)
\end{gathered}
$$

$t_{1}, t_{2}$ : Time ( $t$ ) for two consecutive perihelions

The following figure shows the results of a simulation of the orbital motion of a planet considering:

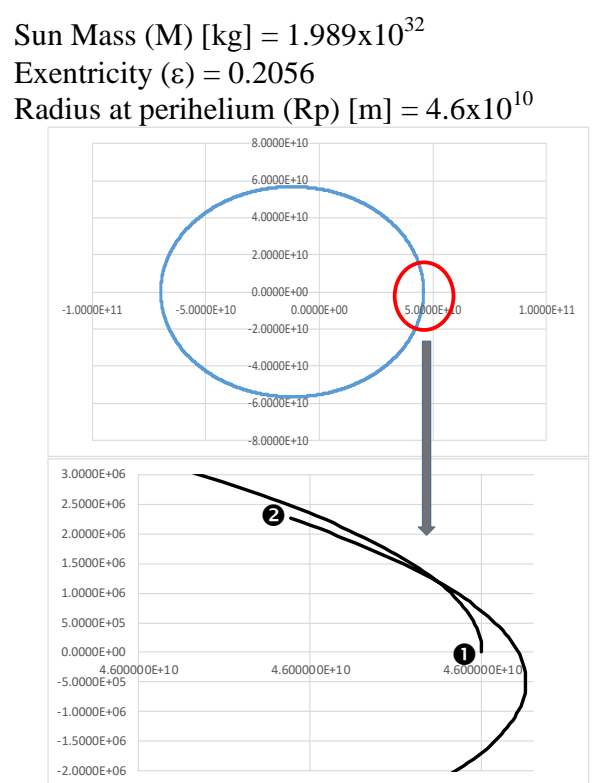

Figure 7: Simulation orbital motion of planets.

D, 2 Two consecutive perihelions 
The $\mathrm{x}, \mathrm{y}$ coordinates of two consequitives periheliums are

$$
\mathrm{x}_{1}, \mathrm{y}_{1}(\mathrm{~m})=46000000000 ; 0.0
$$$$
\mathrm{x}_{2}, \mathrm{y}_{2}(\mathrm{~m})=45999999944 ; 2263829.3
$$

The precession angle $(\delta)$ result:

$$
\delta(\mathrm{rad})=\frac{2263829.3}{46000000000}=4.9214 \times 10^{-5}
$$

The precession angle $(\delta \mathrm{R})$ resulting from the TR (equation (1)) is:

$$
\delta R(r a d)=\frac{3 \times 2 \pi}{(1+\varepsilon) R_{P}} \times \frac{G M}{c^{2}}=5.0202 \times 10^{-5}
$$

The difference between both values is $1.97 \%$

The above results demonstrate that the proposed theory produced similar results than the TR for both circular and elliptical orbits when speed $\left(\mathrm{v} \ll \mathrm{c}_{0}\right)$.

\section{Theory About the Propagation of Light}

The theory that is proposed about the propagation of light in the vacuum assumes, similar to was descrived before for a body in motions, that the SLIDING effect also affects the propagation of light in the vacuum.

Similar to happen for moving bodies, light has two velocities, i) the velocity $\left(\mathrm{c}_{0}\right)$ that determines the photon energy, ii) the velocity (c) that determines the light wavelength. The difference between both velocities is significant only for very long propagation distances (millions of light-years). Velocity (c) is greater than $\left(\mathrm{c}_{0}\right)$, this allows explain the red-shift observed in the light camming from faraway stars.

The proposed theory, considers that the distance traveled by the photon (or the wave) in intervals of time $(\Delta t=1 / f)$ successive is increasing with the distance (D) traveled by the light beam and it is greater than the distance resulting considering the speed of light measured at short distances from the light source ${ }^{2}$.

$$
\begin{gathered}
\lambda_{0}=\frac{c_{0}}{f} \\
\lambda_{1}=\lambda_{0} \times\left(1+K \lambda_{0}\right) \\
\lambda_{2}=\lambda_{1} \times\left(1+K \lambda_{0}\right) \\
\lambda_{n}=\lambda_{n-1} \times\left(1+K \lambda_{0}\right)
\end{gathered}
$$

Where $\mathrm{K}$ is a constant that is determined experimentally.

By integrating, results in the following expression for the wavelength $(\lambda)$ at a distance (D) from the light source:

$$
\begin{gathered}
\lambda_{E}=\lambda_{0} \\
\lambda_{O}=\lambda_{n} \\
\lambda_{O}=\lambda_{E} \times e^{K \lambda_{0} N}
\end{gathered}
$$

$$
\begin{gathered}
D=\int_{0}^{N} \lambda_{E} \times e^{K \lambda_{0} N} d N=\frac{1}{K}\left(e^{K \lambda_{0} N}-1\right) \\
D=\frac{1}{K} \times\left(\frac{\lambda_{O}}{\lambda_{E}}-1\right) \\
\lambda_{O}=\lambda_{E} \times(1+K D) \\
\frac{\lambda_{O}-\lambda_{E}}{\lambda_{E}}=K D
\end{gathered}
$$

The above expression has the same form as Hubble's Law.

$$
\frac{\lambda_{O}-\lambda_{E}}{\lambda_{E}} c_{0}=H_{0} \times D
$$

\section{H0 : Hubble's constant}

The value of $\mathrm{K}$ that complies with experimental observations is:

$$
\begin{gathered}
K\left[\frac{1}{m}\right]=\frac{0.2}{c_{0}^{3}}=\frac{H_{0}}{c_{0}} \\
H_{0}\left[\frac{\frac{k m}{\mathrm{~s}}}{M p c}\right]=\frac{0.2}{c_{0}^{3}} \times \frac{3.0857 \times 10^{22}}{1000}=68.66
\end{gathered}
$$

Once the constant $(\mathrm{K})$ is known, the wavelength increment $(\Delta \lambda)$ observed each $(\Delta t=1 / f)$ result.

$$
\begin{gathered}
\Delta \lambda_{i}=\lambda_{i}\left(1+\frac{0.2}{c_{0}^{2}} \times \frac{1}{f}\right) \\
\lambda_{i+1}=\lambda_{i}+\Delta \lambda_{i}
\end{gathered}
$$

The following figure shows the conceptual design of an experiment that could demonstrate the theory proposed about the propagation of light. In this experiment, two light beams (L1, L2) arrives at the screen. The beam L1 traveled the distance $\mathrm{d} 1$ twice, the beam $\mathrm{L} 2$ traveled the distance $\mathrm{d} 2$ twice. All the components of the experiment are at rest within the same inertial system $(\mathrm{x}, \mathrm{y})$.

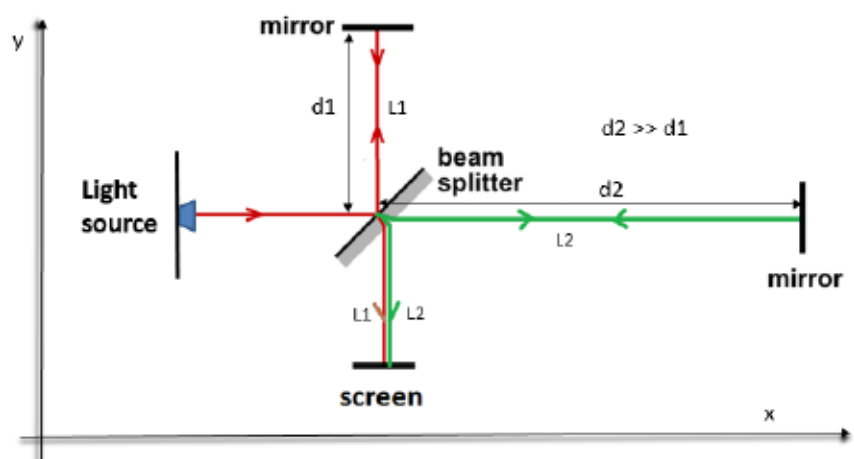

Figure 8: Theoretical experiment to test the wavelength variation of light.

If $\mathrm{d} 2>>\mathrm{d} 1$ what would be observed is that the light beam L2 has a wavelength $(\lambda 2)$ greater than the wavelength $(\lambda 1)$ of the light beam L1.

That is, observers at rest along the light propagation path should be able to measure a wavelength of the light beam that increases with the distance from the light source. It should be noted that the increase in wavelength is not due to

\footnotetext{
${ }^{2} \mathrm{c}_{0}[\mathrm{~m} / \mathrm{s}]=299.772 .450$
}

\section{Volume 10 Issue 12, December 2021}


the Doppler Effect since it is assumed that the light source and the observer are in the same inertial system and at rest to each other.

Under the conditions indicated above, the increase in wavelength due to the propagation of light should be interpreted as an apparent increase in the speed of light (c) due to the SLIDING effect.

$$
\begin{aligned}
& c_{1}=\lambda_{1} \times f \\
& c_{2}=\lambda_{2} \times f
\end{aligned}
$$

Equation (7) allows us to determine the variation of apparent light speed along the propagation path, where $(\mathrm{t})$ is the time elapsed from light emission.

$$
\begin{gathered}
N=\frac{c_{0}}{\lambda_{0}} \times t \\
D(t)=\frac{1}{K}\left(e^{K c_{0} t}-1\right) \\
c(t)=\frac{d D(t)}{d t}=c_{0} \times e^{\frac{0.2}{c_{0}^{2}} \times t} \\
c(D)=c_{0} \times\left(1+\frac{0.2}{c_{0}^{3}} \times D\right)
\end{gathered}
$$

The foregoing does not contradict the experiments of Michelson and Morley since the proposed increase in the wavelength is observable only for very large propagation distances (millions of light-years) ${ }^{3}$.

\subsection{Observable Universe (The Olbers's Paradox}

As previously mentioned, the proposed theory considers that as the light propagates from the emission source increases its wavelength progressively keeping its energy invariant.

This implies therefore that there will be a horizon beyond which we will not be able to observe a star since the light that comes to us from it will no longer be in the spectrum of visible light. If this theory is true, the light of a star that is close to this horizon should be seen as red.

This horizon is a circle centered on us with radius RO given by the following expression:

Where:

$$
R O=\frac{c_{0}^{3}}{0.2} \times\left(\frac{\lambda_{\mathrm{M}}}{\lambda_{\mathrm{m}}}-1\right)
$$

$\lambda_{\mathrm{M}}: 780 \mathrm{~nm}$ (Maximum wavelength of the visible light spectrum)

$\lambda_{\mathrm{m}}$ : Minimum wavelength of the Star Radiation Spectrum

For example, for a star with a temperature similar to our sun $\left(\lambda_{\mathrm{m}}=300 \mathrm{~nm}\right)$ result

\section{$\mathrm{RO}=22796$ millions of light-years}

The time (T) elapsed since the light left the star until it reaches us is given by the following expression:

\footnotetext{
${ }^{3}$ A light beam that travels across our galaxy $(100,000$ lightyears) experiences a wavelength increase of only $0.0007 \%$
}

$$
T=\frac{c_{0}^{2}}{0.2} \times \ln \left(\frac{\lambda_{\mathrm{M}}}{\lambda_{\mathrm{m}}}\right)
$$

For the previous example, time $\mathrm{T}$ result

$$
T=13614 \text { millions years }
$$

For each star there is a value of RO with higher values the higher the temperature of the star. Therefore there will be always a horizon that determines the stars that are visible from the earth. Light from stars located beyond this horizon will reach us with wavelengths outside the spectrum of visible light (infrared). The sky is black because we can not see all the stars in the universe avoiding, therefore, the Olbers paradox.

The increase of observed wavelength can be interpreted as a reduction of the temperature $\left(\mathrm{T}_{\mathrm{O}}\right)$ of the observed radiation received from a faraway star $\left(\mathrm{T}_{\mathrm{O}}<\mathrm{T}_{\mathrm{E}}\right)$. The Planck's Law for the radiation of a black body including the distance effect due to the SLIDING effect is as follows.

$$
\begin{array}{r}
R\left(\lambda_{\mathrm{O}} ; T_{\mathrm{O}}\right)=\frac{2 h f c_{0}}{\lambda_{O}^{4}} \times \frac{\left(1+\frac{0.2}{c_{0}^{3}} \times D\right)^{4}}{\left(e^{\frac{h f}{K_{B} T_{O}}}-1\right)} \\
T_{\mathrm{O}}=\frac{T_{\mathrm{E}}}{\left(1+\frac{0.2}{c_{0}^{3}} \times D\right)}<T_{\mathrm{E}}
\end{array}
$$

As an example, the following figure shows the observed radiation received from a star similar to our sun $\left(\mathrm{T}_{\mathrm{E}}=5777 \mathrm{~K}\right)$ located at a distance near to RO.

The observed radiation is similar to the radiation of a black body with a temperature of $2224 \mathrm{~K}$. A minimum portion of the observed radiation is within the visible light spectrum. The total energy don't change.

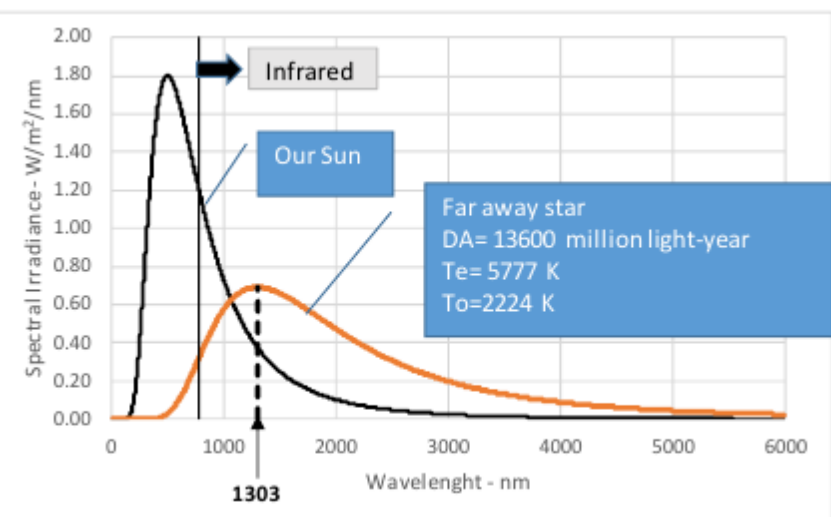

Figure 9: Observed radiation received from a far-away star.

\subsection{Gravitational Effects}

Experiments carried out by Sir Arthur Stanley Eddington in 1919, and more recently, observing the light coming from Quasar 3C279, which is blackened by the sun every October,

\section{Volume 10 Issue 12, December 2021}


show that light does not propagate in a straight line, curving in the presence of strong gravitational fields (the stars) ${ }^{4}$.

The theoretical considerations developed in this document allow us to predict this behavior considering that the light that propagates towards stars has different speeds depending on the direction of propagation (tangential, radial) to the star.

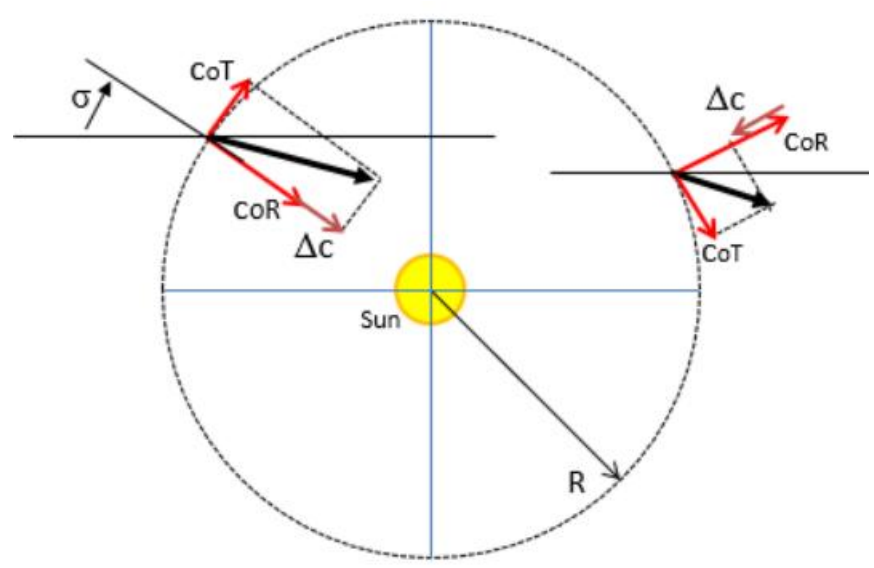

Figure 10: The radial component of light speed $\left(c_{0} R\right)$ changes due to the gravitational field of the star.

In the tangential direction, the speed of light is not affected by gravitation field, instead, in the radial direction the speed of light results from the following expression:

$$
\begin{gathered}
c_{0 T}=c_{0} \sin (\sigma) \\
c_{0 R}=c_{0} \cos (\sigma) \\
c_{T}=c_{0 T} \\
c_{R}=c_{0 R}+\Delta \mathrm{c} \\
\Delta c=\frac{c_{0} \frac{R_{0}}{R}}{1-\frac{R_{0}}{R}}=\frac{c_{0}}{\frac{R}{R_{0}}-1} \\
R_{0}[m]=K_{L} M \\
K_{L}[\mathrm{~m} / \mathrm{kg}]=2.0 \times 10^{-25}
\end{gathered}
$$

where
G: Gravitational constant
R: Distance to the star
M: Solar mass

The difference $(\Delta c)$ between the light speed $c R$ and $c_{0} R$ produces a deflection of the light beam that passes near to a star as shown in the following figure for a light beam passing near to our sun.

\footnotetext{
${ }^{4}$ According to these last observations, the light beam rotates an angle $(\delta=1.73 \pm 0.05 \operatorname{arcsec}$
}

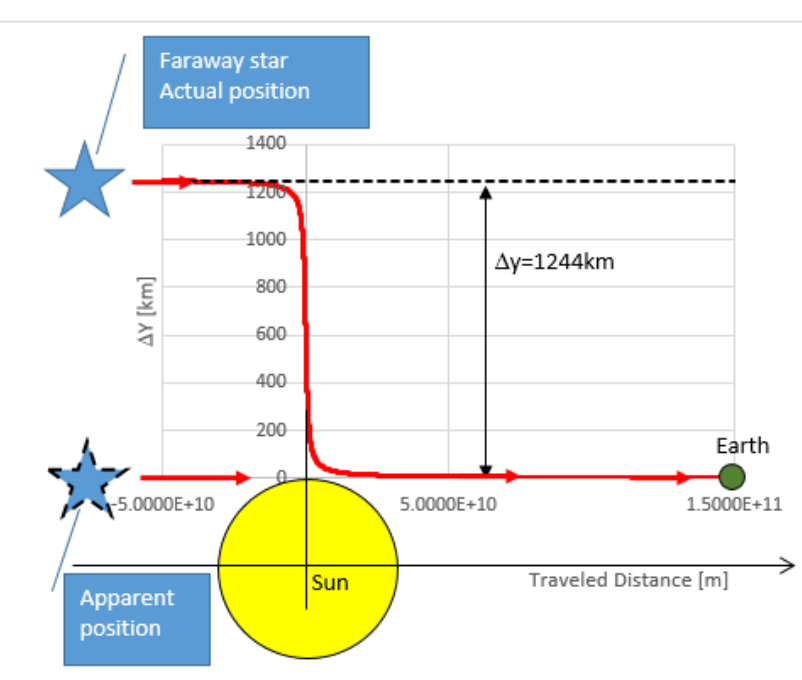

Figure 11: Deflection of a light beam passing over the surface of the sun.

In this case, the deflection $\Delta y[\mathrm{~km}]=1244$ equivalent to an angle $\delta=1.71$ arcsec, value that is similar to measured value (1.73 $\operatorname{arcsec})$.

Equation (9) shows that for $\mathrm{R}=2 \mathrm{R}_{0}$ result

$$
\begin{gathered}
\Delta c=c_{0} \\
c_{R}=0.0
\end{gathered}
$$

This means that, for a star with radius $\mathrm{R}=2 \mathrm{R}_{0}$ the outcoming light beam has a radial light speed $\mathrm{cR}$ equal to zero, meaning that light cannot propagate and, consequently, we can not see the star. For our sun, $2 \mathrm{R}_{0}=795.6 \mathrm{~km}$, so if all the mass of our sun is compressed into this radius we couldn't see our sun (it is what we have called a "black hole".

\section{Conclusions}

Within the framework of the proposed theory, we can conclude that the propagation of energy through the vacuum, either in the form of a light beam or a moving body with mass (m), produces the SLIDING effect.

Is, therefore, the SLIDING effect the reason why we observe the "red-shift" in the spectral lines that they reach us from distant stars and the perihelion's precession in the orbit of the planets, among other facts.

If the red-shift is the result of the SLIDING effect, that implies that our universe is not expanding, therefore, we should consider that there was no initial explosion (Big Bang), that the universe has always existed and will exist forever.

The universe has a "horizon" only because the light that reaches us from a star located beyond that horizon has a wavelength outside the visible spectrum (infrared radiation) as a result of the sliding that increases its wavelength as the light propagate.

We need to review our understanding of how the universe works. Some of the relevant topics are:

\section{Volume 10 Issue 12, December 2021}


1. The concept of time. Bodies move a distance greater than that foreseen by Newton's Laws, not because time goes by slower for them but because it is the body that, due to sliding, travels a greater distance at the same time.

2. Gravity. If the precession of the planets is the consequence of the SLIDING effect, so the idea that explains gravity as a curvature of the "space-time" possibly be not valid.

3. The origin of matter. If the universe has existed forever and will exist forever, the question arises about the origin of hydrogen. The stars are formed by the concentration of hydrogen atoms by the effect of gravity. The stars then, throughout their life and death, converts hydrogen into heavier atoms (the other elements we know). This implies that there should be a "factory" of hydrogen. We have not found so far how this hydrogen factory works and therefore what is the origin of the star fuel.

4. Theory about the Light. Michelson's experiments led Einstein to consider as an axiom that the speed of light in the vacuum is constant. If the proposed theory about the red-shift is correct, it implies that the speed of light is modified as it propagates through the vacuum. The discussion on the nature of light, wave/particle, also remains. To reconcile all these aspects of light, a new theory about the nature of light that explains the observations will be necessary.

Acknowledgments: To my family, to La Plata University, Argentina.

\section{References}

[1] Sir Isaac Newton 1687. The mathematical principles of natural philosophy.

[2] A. A. Michelson and E. W. Morley 1887. On the Relative Motion of the Earth and the Luminiferous Ether. The American Journal of Science

[3] Einstein, A. 1905 - Zur Elektrodynamik bewegter Körper. Annalen der Physik.

[4] Einstein, A. 1916 Die Grundlage der allgemeinen Relativitatstheorie. Annalen der Physik.

[5] Edwin Hubble 1929 A relation between distance and radial velocity among extra-galactic nebulae. PNAS

[6] A. A. Penzias y R. W. Wilson 1965 A Measurement of Excess Antenna Temperature at $4080 \mathrm{Mc} / \mathrm{s}$. Astrophysical Journal

[7] Rainville, S. et al. 2005 A direct test of $\mathrm{E}=\mathrm{mc}^{2}$. Nature

[8] Marcelo Samuel Berman 2007 Introduction to General Relativistic and Scalar-Tensor Cosmologies.

[9] Stanford Encyclopedia of Philosophy 2012 The Equivalence of Mass and Energy.

[10]Clifford M. Will 2014 Confrontation between General Relativity and Experiment. Department of Physics University of Florida Gainesville FL 32611, U.S.A.

Volume 10 Issue 12, December 2021 